\title{
Session based cooperation in cognitive radio networks: network level approach
}

\author{
Sunil Ghildiyal ${ }^{1}$, Himanshu Goel ${ }^{2}$, Anupam Semwal ${ }^{3}$ \\ ${ }^{1,2}$ Uttaranchal University, India \\ ${ }^{3}$ Sainath University, India
}

\begin{tabular}{|c|c|}
\hline Article Info & ABSTRACT \\
\hline Article history: & \multirow{9}{*}{$\begin{array}{l}\text { The cognitive radio prototype performance is to alleviate the scarcity of } \\
\text { spectral resources for wireless communication through intelligent sensing } \\
\text { and quick resource allocation techniques. Secondary users (SU's) actively } \\
\text { obtain the spectrum access opportunity by supporting primary users (PU's) in } \\
\text { cognitive radio networks (CRNs). In present generation, spectrum access is } \\
\text { endowed through cooperative communication-based link-level frame-based } \\
\text { cooperative (LLC) principle. In this SUs independently act as conveyors for } \\
\text { PUs to achieve spectrum access opportunities. Unfortunately, this LLC } \\
\text { approach cannot fully exploit spectrum access opportunities to enhance the } \\
\text { throughput of CRNs and fails to motivate PUs to join the spectrum sharing } \\
\text { processes. Therefore, to overcome this con, network level cooperative (NLC) } \\
\text { principle was used, where SUs are integrated mutually to collaborate with } \\
\text { PUs session by session, instead of frame based cooperation for spectrum } \\
\text { access opportunities. NLC approach has justified the challenges facing in } \\
\text { LLC approach. In this paper we make a survey of some models that have } \\
\text { been proposed to tackle the problem of LLC. We show the relevant aspects } \\
\text { of each model, in order to characterize the parameters that we should take in } \\
\text { account to achieve a spectrum access opportunity. }\end{array}$} \\
\hline Received Mar 06, 2019 & \\
\hline Revised Sep 12, 2019 & \\
\hline Accepted Oct 20, 2019 & \\
\hline Keywords: & \\
\hline & \\
\hline Cooperative communication & \\
\hline Integrated & \\
\hline Spectrum access & \\
\hline
\end{tabular}

This is an open access article under the CC BY-SA license.

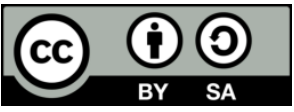

\section{Corresponding Author:}

Sunil Ghildiyal,

Uttaranchal University,

Arcadia Grant, Chandanwari, Premnagar, Dehradun, Uttarakhand 248007, India.

Email: Sg124ddn@gmail.com

\section{INTRODUCTION}

In recent times Cooperative communications have achieved considerable importance. With the major focus on physical layer performance, Cooperation can happen at different communication layers [1], [2]. In that point, collaboration advantages are undeniable, since the investigated frameworks regularly have a place with a solitary entertainer with enthusiasm to boost a particular utility [3]. The advantages of making use of cooperative techniques in improvising system performance were found to be multifold in the recent times, primarily by focusing on network layer. The improvement in performance could be with respect to data [4] consistency [5] and time lagging [6]. Cognitive radio allows secondary users to actively support with primary users' relays to achieve spectrum access opportunities as an incentive [7]. Positively there exist two approaches on cognitive radio networks, there are: a) Common model: In this framework secondary user's activity is nullified by the primary users. In turn secondary users detect the radio signaling environment in search of bandwidth while primary users are not active for utilizing the channel and then make use of identified transmission opportunity. b) Spectrum lease/cooperation-based spectrum: here secondary users are actively involved by assisting primary users to achieve spectrum access opportunities as a reward [8]. 
With the help of relay channel, information theory has led the way to plan for cooperative communications. Therefore, relay channel stands as the fundamental pedestal for practical implementation of cooperative communication. This cooperative communication has been extensively accepted toafford advanced communication levels and consistency in wireless networks with time change channel [2]. This was proposed in the early times by Van der Meulen [9], and it was described and characterized in detail in [10]. In recent times, relay channel has been acknowledged widely in the field of wireless networks. In [11] on the study of a typical relay channel a procedure was illustrated for choice of choosing recipient and transmitter time slots depending on the suitable conditions and based on the worth of the links involved. In the study [12], related to full and half duplex relaying, it says if the number of antennas are equal at the source and relaying point, or if the number of antennas are higher at destination point than source, then half duplex achieves higher throughput rates when compared with full duplex relaying, and this is possible only in few cases but not all.

The study [13] put forward two buffer mediated relay selection plans using beam forming and inter relay interference. In the study [14] where Interference limitation was deployed to allow selected set of relays activated to get the most out of the average capability of the network. For multiple relay stations (RS) with adaptive relay station (RS) activation, a rationalized scheme of DMA based cellular resource provision schemes were estimated [15]. As mentioned, the majority of the works in this area aim on potential gains by cooperation at the physical layer. Recent works [4] and [15] indicates that same gains can be achieved by network-layer cooperation. By network-layer cooperation they consider relaying to be taking place at a protocol level avoiding physical layer considerations. Random multiple access schemes in these works use the collision channel model with erasures, where there are more chances of failure of concurrent transmissions [15-17]. The collision channel however is not the appropriate model for wireless networks. A survey has been undertaken on network-level session-based cooperative (NLC) approach for CRNs so that the spectrum access opportunities are utilized more efficiently. Unlike the LLC approach where SUs cooperates with PUs for their own spectrum access opportunities, in NLC approach, SUs is grouped together and cooperate with PUs for the spectrum access opportunities of the corresponding group.

\section{PRIMARY (PU) AND SECONDARY USERS (SU) NETWORKING: PUS AND SUS ROLE}

In cognitive radio networks, there exist three spectrum sharing methods.

a. Underlay: In underlay, SUs can convey in parallel with the PU only if the hindrance level from SU to PU is less than some limit. Threshold is the maximum level of intrusion that PU can withstand without causing any hindrance the intended data rate or intended SNR/SNIR. The PU does not care about the existence of SU. It only cares about the interference level.

b. Overlay: In overlay, there is a mutual cooperation between PU and SU. So, PU knows the existence of SU. SU may act as a cooperative relay for the PU. In return of participation, SU get the opportunity to get to the channel with PU in view of superposition coding or non-orthogonal numerous accesses.

c. Opportunistic: In this method, SU can access the channel only if there is no primary transmission. That means, SU has to monitor the channel to know the existence of PU. Here, the duty goes to the SU totally. There are different methods such as energy detection, cyclostationary detection, matched filter and waveform bases spectrum detection etc. to detect the existence of PU. Moreover, hybrid spectrum sharing consists of the combination of above methods. In addition to the above methods: In Spectrum leasing protocol, PU leases or offers a segment of its time slots or range to the SU. It depends on the mutual understanding.

\subsection{Role of energy consumption in designing the communication strategies}

Energy conservation is also one major factor in articulating the designing cooperative schemes since it has direct effect on users' communication strategies. In this view, many stratagies, based upon energyaware cooperative schemes are investigated based on a sum-constrained power allocation game and a power control game by introducing intermediate users (IU's). These intermediate usersassist withPrimary Users in rush movement transmission and contribute to the spectrum access opportunities with the enduring Secondary users. Since SUs of limited power; PUs may need to recruit multiple SUs to relay their data in a multihop fashion over the desired long distances. In, this multihop relay selection problem is observed and studied based on a network formation game. As motivated by serious and increasing concerns on information security, two types of cooperative schemes are proposed to improve PUs' secrecy and data rate [14].

Implementation of choosing Intermediate User is done by highest biased bipartite identical algorithm and, cooperating pairs use is improved from the relation of collaboration pair use to the whole energy expenditure considering Intermediate Users (IU) efficiency. In addition, with the collaboration between intermediate users and secondary users, the secondary users get an opportunity of transmission to 
primary users with less energy consumption. At the same time intermediate users have full role to play and their communication consistency can also be increased. [18].

\subsection{Session based cooperation framework in cognitive radio networks}

A wireless system is of limited spectrum; the ever-increasing channel or bandwidth capacity demands result in the spectrum crunch. A supplementary network infrastructure was also engaged to behave as base stations (BSs), an effective move to improve the scarcity of spectrum [1]. Thus, small cell BSs (SCBSs) will have to be widely deployed. SCBSs have a capability of providing high network for wireless users by having close proximity to the users. However, a SCBS also has a limited area of coverage. As an effect, the overall energy utilization of cellular networks will increase on continuous basis [2]. Thus, existing wireless access networks are in due course stressed by the spectrum lack and energy consumption. It is advantageous to merge spectrum and energy harvesting technologies to free the wireless networks from these limitations. Energy harvesting techniques like BSs allow network devices to produce electricity from renewable energy sources, and sustainable bio fuels.

To provide communication services to SUs, the SSP deploys or leases some BSs for fundamental service coverage as done in cellular systems and CR routers for efficient resource utilization. Base stations are interrelated with wired links via Internet or further high-speed information networks and work as gateways for $\mathrm{CR}$ routers, so that the $\mathrm{CCHN}$ can gain backbone network services. Base stations and CR routers both are well furnished with many radio interfaces. They are equipped with interfaces like cellular interface, cognitive radio interface, and Wi Fi interface, and can operate over the SSP's basic bands, unlicensed bands (e.g., ISM bands), and unoccupied licensed bands. PUs and SUs are consistently circulated in a CCRN. The information has been conveyed to the BS under its own authorized channel by a base station (BS). BS serve PU, given that the ranges of PUs are orthogonal in rate of recurrence as well as space. Access points (APs) coincide in a similar range serving SUs and each SU speaks with its relating AP. The main stage collaboration is between the PU and the chosen group head IU, while the second stage participation is between the group head and different SUs in the group.

The collaboration amongst SUs and PUs happens in a two-stage participation plot in each scheduled slot. The accomplice IU choice plan is first executed, and after that the group head IU participates with the PU in a TDMA way that the PU conveys its bundle to IU and afterward IU impart PU's last slot to the BS parallel. Subsequent to PU and IU collaboration, SUs additionally joins IU who form a gathering from the encompassing anticipating SUs. At that point, the IU and the SUs in the group coordinate by cooperative system coding.The SUs, who take an interest in the participation with the PUs, send inputs with them transmit control value by conveying PUs activity to the BS. Keeping in mind the end goal to enhance the execution of primary network, the BS communicates the participation choice prerequisite to its encompassing SUs. On the off chance if one SU can fill in as the transfer for numerous PUs, it sends diverse transmit power value to every PU to the BS. In any case, in genuine systems, some SUs would not willing to participate with the PU, because SUs need more energy to convey information to PUs and the energy they gain is very less. That is the proportion of utility is more than supply. However, sometimes the SUs still wants to pick up the auxiliary transmission modes in order to enhance their utility. Keeping in mind the end goal to tackle the previously mentioned issue, the chosen IU coordinates with the rest of the SUs to profit them. Thus, through the participation between group head IU and different SUs in the bunch, the IU can enhance its own particular execution also.

The time span configuration incorporates two collaborations: the principal stage participation and the second stage collaboration. In the IU choice time of the primary stage collaboration, after BS gets the affirmation and the data from potential IUs, the BS exploits the greatest weighted bipartite coordinating calculation to locate the most suitable helpful SUs, i.e., the IUs. After accomplice IU choice, the PU collaborates with the IU in a TDMA way. At that point, the IU communicates its participation prerequisite to start the second stage collaboration. The SUs sends the affirmation that they need to join into the participation with the IU. From that point onward, the IU transmits its bundle towards the related AP. Amid this transmission, the channel conditions are thought to be steady amid a fix schedule slot, however fluctuate autonomously starting with one slot then onto the next. The spectrum sharing system works in a time slotted way and transmission channels are expected to fit in with a Rayleigh flat fading model. The CSI is accessible, which is assessed by accomplishing strategies, for example, minimum mean-square-error (MMSE) estimation, least squares (LS) estimation and [19]. Process, the encompassing SUs (frame a bunch) who are associated with the collaboration can catch the information.

Then, with use of network coding, the SUs creates new combinations of packets from the received packets and transmit those towards the respective AP within or out of custer as needed. Cooperation scheme between the cluster head IU and SUs in the cluster is referred as cooperative network coding, where IU is the source and the corresponding AP is the destination, and then SUs forms a cluster to help IU relay the data 
from the source to the destination. Care of energy efficiency is highly considered while designing the system by using a ratio of utility to energy, which enables a tradeoff between optimized consumption of utility and energy. IU selection is performed to select the IUs who cooperate with the PUs. The IUs are a group of SUs that have better channel conditions than other SUs to relay PUs' traffic.

\section{DESIGN OF COGNITIVE CAPACITY HARVESTING NETWORK: BUILT FEATURES OF CCHN}

The CCHN comprises of a BSs, SSP, CR switches and SUs [20]. The SSP is a autonomous wireless service organizer, for example, a cellular administrator that will give better or new sorts of administrations to cellular clients, and has its own particular authorized range groups, alluded to as the SSP's fundamental groups. The SSP is responsible for service provision and spectrum coordination within its area of coverage. To provide communication services to SUs, the SSP deploys or leases some BSs for fundamental service coverage as done in cellular systems and CR routers for efficient resource utilization. BSs are interrelated with wired associations through Web or other rapid information systems and work as passages for CR routers, so that the CCHN can pick up important network administrations. BSs also serve as an operator for the SSP to trade control motioning with CR routers and SUs. CR routers are intelligent wireless routers with cognitive capability and operate under the supervision of the SSP. Both BSs and CR switches are furnished with various radio interfaces, for example, cell interface, cognitive radio interface, and WiFi interface, and can operate over the SSP's basic bands, unauthorized bands (e.g., ISM bands), and unoccupied authorized bands. The CCHN architecture radio mesh network to help the SSP deliver services to SUs in collaboration with BSs. SUs are wireless gadgets like laptops and smart phones obtaining operations via certain access technologies (e.g., GSM/GPRS, LTE and WiFi) and may or may not have cognitive capability. SUs gets to the SSP's administrations by associating with CR routers or BSs, and CR switches directly connecting to SUs are called edge $\mathrm{CR}$ routers.

In the event that SUs had cognitive ability, they can speak with edge CR routers through both their fundamental access innovations and cognitive radio. If SUs' devices do not have cognitive radio interfaces, edge CR routers will tune to the interfaces which SUs normally use to deliver services. Each edge CR router constantly collects information appeal in its scope range and present those collected data appeals to SSP for resource allocation. Based on the data requests and available resources, the SSP carries out network optimization, and the decisions will be delivered to CR routers via the SSP's basic bands. Under the direction of the SSP, BSs and CR switches aggregately develop ways to convey administrations to SUs through multihop transmissions. As shown in [20], the CCHN architecture is very flexible in supporting various schmes of spectrum-sharing paradigms, including spectrum-sensing based and spectrum-auction-based spectrum sharing. In this paper, we consider the use of the CCHN to support another paradigm, i.e., cooperation-based spectrum sharing.

\section{BASIC NETWORK MODELS WITH THEIR EFFECTIVENESS CRITERIA}

\subsection{Cooperative cognitive network model}

In the cognitive radio system, two networks exist, primary network and amplify-and-forward (AF) cooperative communication secondary network. The stream of information is essential and its path isprimary user passes on to a primary destination (PD), and secondary transmitter (ST) passes on its information to a secondary destination (SD) at the comparable time. In order to implement the cooperation, 'receive diversity protocol' is exhibited [4]. The transmitters, destinators and relay terminals all contain one antenna and one transmit. In early stage, relay and SD gets information from ST. At that point in the second stage, the ST remains inert, while the relay increases and transfers the information. In CRS where there is no spectrum sense action, the convey power of ST should be restricted to uphold a preferred QoS of PT.PT convey the signal xp to PD where the transmit energy of PT is signified by PPT and information rate by Rp. Similarly, ST pass on the signal xs to SD with transmit energy PST. The loss of primary transmission is restricted by threshold PT hr, to accomplish the coveted QoS of primary transmissions [20]. Cooperative cognitive network model as shown in Figure 1. 


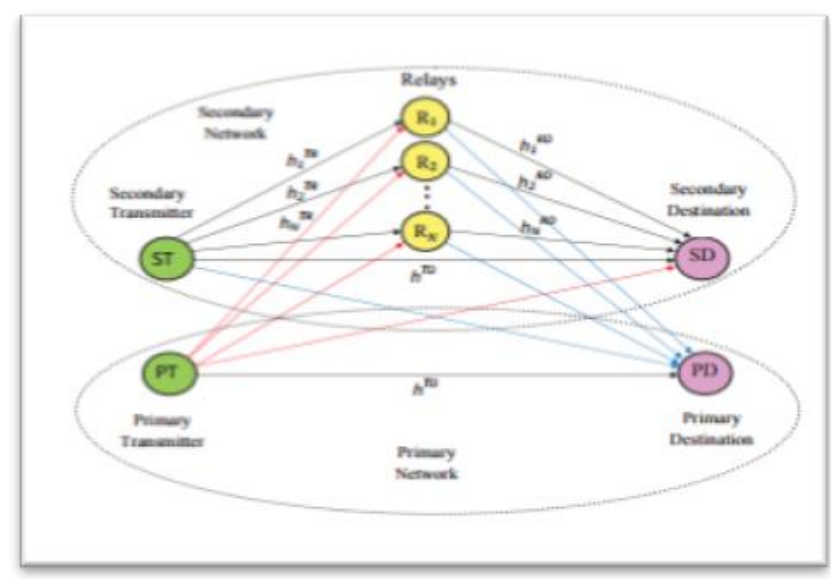

Figure 1. Cooperative cognitive network model

\subsection{Constraints with respect to communication}

\subsubsection{Flow route and link schedule constraints}

To optimally utilize network resources, we should mutually consider flow route and connection schedule which are tightly coupled problems. On the one hand, the scheduling at the data link layer should ought to have the capacity to support the flow at the system layer. On the other hand, how much flow can be carried at the system layer is controlled by the planning at the data link layer.

\subsubsection{Link scheduling constraints}

In this paper, we consider time-based link scheduling where different links are allocated with certain periods of time to build up flows between end systems. Consequently, the flow rates which the network layer can provide depend on the data rate of each link as well as the time share allocated to these links. To give PUs with motivations to collaborate, the SSP should ensure PUs' data is delivered to the destination earlier than what would have been scheduled without the SSP's help.

\section{CONCLUSION}

With this study we can conclude saying there are numerous benefits of integrating a cooperative spectrum sensing system inside a cognitive radio network, which are: a)Significant reduction in Hidden node problem: One of the main issues with LLC is that despite the fact that the cognitive radio network would not recognize an PU transmitter, it mediates with the recipients which have capacity to distinguish both the PU and cognitive radio framework transmissions. By utilizing a cooperative detecting framework, it is conceivable to diminish the likelihood of this feature because a prominent number of recipients will have the capacity to develop an exact image of the transmissions in the area. b) Agility Improvement: By cooperation spectrum sensing nodes increases the detecting to be more acceptable and improved choice for channel moves can be processed, inturn providing an increase in agility. c) Decrease of false alarms: Presence of multiple nodes exhibiting the spectrum sensing, channel signal identification is more precise and these reduce the number of false alarms. d) Precise signal detection: Accurate signal identification and reliability of complete system is possible with cooperative spectrum.

\section{REFERENCES}

[1] G. Kramer, I. Maric, R. D. Yates, "Cooperative communications," Found. Trends Netw., vol. 1, pp. 271-425, 2006.

[2] J. Laneman, D. Tse, and G. W. Wornell, "Cooperative diversity in wireless networks: Efficient protocols and outage behavior," IEEE Transactions on Information Theory, vol. 50, pp. 3062-3080, 2004.

[3] T. Ng and W. Yu, "Joint optimization of relay strategies and resource allocations in cooperative cellular networks," IEEE Journal on Selected Areas in Communications, vol. 25, pp. 328-339, Feb 2007.

[4] A. Sadek, K. Liu, and A. Ephremides, "Cognitive multiple access via cooperation: Protocol design and performance analysis," IEEE Transactions on Information Theory, vol. 53, no. 10, pp. 3677-3696, 2007.

[5] K. J. R. Liu, A. K. Sadek, W. Su, and A. Kwasinski, "Cooperative Communications and Networking," Cambridge University Press, 2009.

[6] N. Pappas, M. Kountouris, A. Ephremides, and A. Traganitis, "Relay-assisted multiple access with full-duplex multi-packet reception," IEEE Transactions on Wireless Communications, vol. 14, no. 7, pp. 99, 2015. 
[7] S. Haykin, "Cognitive radio: brain-empowered wireless communications," IEEE J. Sel. Areas Commun., vol. 23, no. 2, pp. 201-220, Feb 2005.

[8] O. Simeone, I. Stanojev, S. Savazzi, Y. Bar-Ness, U. Spagnolini, and R. Pickholtz, "Spectrum leasing to cooperating secondary ad hoc networks," IEEE J. Sel. Areas Commun., vol. 26, no. 1, pp. 203-213, Jan 2008.

[9] E. C. V. D. Meulen, "Three-terminal communication channels," Advances in Applied Probability, vol. 3, no. 1, pp. $120-154,1971$.

[10] T. Cover and A. Gamal, "Capacity theorems for the relay channel," IEEE Transactions on Information Theory, vol. 25, pp. $572-584$, Sep 1979.

[11] N. Zlatanov, R. Schober, and L. Lampe, "Buffer-aided relaying in a three-node network," in IEEE International Symposium on Information Theory Proceedings (ISIT), pp. 781-785, Jul 2012.

[12] N. Zlatanov and R. Schober, "Buffer-aided half-duplex relaying can outperform ideal full-duplex relaying," IEEE Communications Letters, vol. 17, pp. 479-482, Mar 2013.

[13] S. M. Kim and M. Bengtsson, "Virtual full-duplex buffer-aided relaying - relay selection and beamforming," in 24th IEEE International Symposium on Personal Indoor and Mobile Radio Communications (PIMRC), pp. 1748-1752, Sep 2013.

[14] N. Nomikos, T. Charalambous, I. Krikidis, D. Skoutas, D. Vouyioukas, and M. Johansson, "Buffer-aided successive opportunistic relaying with inter-relay interference cancellation," in 24th IEEE International Symposium on Personal Indoor and Mobile Radio Communications (PIMRC), pp. 1316-1320, Sep 2013.

[15] B. Rong and A. Ephremides, "Cooperation above the physical layer: The case of a simple network," in IEEE International Symposium on Information Theory (ISIT), pp. 1789-1793, Jun 2009.

[16] N. Pappas, A. Traganitis, and A. Ephremides, "Stability and performance issues of a relay assisted multiple access scheme," in IEEE Global Telecommunications Conference (GLOBECOM 2010), pp. 1-5, Dec 2010.

[17] N. Pappas, M. Kountouris, A. Ephremides, and A. Traganitis, "On the stability region of a relay-assisted multiple access scheme," in IEEE Information Theory Workshop (ITW), pp. 1-8, Sep 2013.

[18] Ashish kumar sinha, Rajeev thakur, "spectrum sharing technique for cooperative cognitive radio networks using bipartite graph", International Journal of Computer Applications, vol. 157, no. 9, 2017.

[19] M. Biguesh and A. B. Gershman, "Training-Based MIMO Channel Estimation: A Study of Estimator Tradeoffs and Optimal Training Signals," IEEE Transactions on Signal Processing, vol. 54, no. 3, pp. 328-339, Mar 2006.

[20] T. Jing, S. Zhu, H. Li, X. Xing, X. Cheng, Y. Huo, R. Bie, and T. Znati, "Cooperative relay selection in cognitive radio networks," IEEE Trans. Veh. Technol., vol. 64, no. 5, pp. 1872-1881, May 2015. 